\title{
A MICROELECTRODE ARRAY FOR REAL-TIME NEUROCHEMICAL AND NEUROELECTRICAL RECORDING IN VITRO
}

\author{
Timothy D. Strong ${ }^{a, b}$, Hal C. Cantor ${ }^{a}$, Richard B. Brown ${ }^{b}$ \\ ${ }^{a}$ Advanced Sensor Technologies, Inc., Farmington Hills, MI 48334 \\ ${ }^{b}$ Department of Electrical Engineering and Computer Science, \\ The University of Michigan, Ann Arbor, MI 48109
}

\begin{abstract}
The development of a silicon neural recording device capable of measuring neurochemical and neuroelectrical activity in tissue culture is described. The device consists of a microfabricated array of platinum and chloridized silver electrodes on a silicon substrate. An affixed culture chamber on the surface of the device contained the culture media. Neuron cultures survived for over 75 days in the devices, generating a great deal of neuroelectrical and neurochemical data. The performance of the devices allowed for the study of single neuron interactions with the ability to correlate electrical signals directly with a chemical response.
\end{abstract}

\section{INTRODUCTION}

The understanding of communication between neurons is key to the advancement of neuroscience. It is important for not only purely scientific reasons, but may provide assistance in studying various neurological injuries and disorders, and possibly lead to treatments.

Miniature silicon neurosensors have been available to neuroscientists for many years [1-7]. These devices have enhanced the study of neural signaling significantly. Arrays of sites can be easily produced on such sensors, allowing the study of multiple groups of neurons concurrently. In addition, the silicon substrate enables the inclusion of active electronics, providing low noise, onchip amplification, and multiplexing to reduce lead count. Silicon micromachining techniques allow the final shape of the devices to be tailored to the application, from fine needle-like probes to threedimensional arrays. To date, these devices have provided neuroelectrical signals but no chemical data.

Carbon fibers affixed in pulled glass micropipettes are among the most common devices for neurochemical sensing. The pipettes are drawn under heat and broken, exposing the carbon fibers at the tip, which form the electrode $[8,9]$. Electronics can be used to allow the detection of both electrical and chemical signals. Unfortunately, their manufacture makes it difficult to insure consistent performance. It is also difficult to create an array, since the sensors are manufactured individually. Amplification must be performed using discrete components, requiring long interconnects that are subject to noise.

The neural array described here combines the advantages of both the silicon neurosensors and the carbon fiber electrodes, while eliminating most of the complications. The devices are fabricated using standard silicon processing technology. This provides high yield and consistent performance due to the tight dimensional control of photolithography. Large sensors arrays facilitate the study of snall neural systems in addition to individual neurons. Using these devices, real-time neurochemical and neuroelectrical activity of the neurons can be recorded. This provides the important capability of studying the correlation of electrical and chemical events in neuronal communication.

\section{VOLTAMMETRY IN NEUROSENSORS}

Many physiologically important neurotransmitters, including dopamine and serotonin, are electroactive. The application of a potential will cause electrons to be removed from the species of interest as it undergoes electrochemical oxidation. The observed current is related to the concentration of the species.

In voltammetry, a potential is ramped between two electrodes (usually referred to as a working and a reference electrode) while the current is recorded. As the oxidation potential of each electroactive species is reached, a corresponding current increase will be observed. Adding a third electrode, called an auxiliary electrode, increases sensitivity. With careful circuit design, the current can be made to flow between the working and auxiliary electrodes. The potential is measured between the reference and the working electrodes with little current being carried by the reference electrode. Placement of the reference electrode close to the working electrode removes the effect of differences in solution conductivity from culture to culture [10]. A single reference electrode can be shared among several sensing sites.

Cyclic voltammetry (CV) extends the voltage sweep by reversing the potential and causing the newly oxidized species to undergo reduction. The reduction cycle provides additional information for the identification of species and also mediates some of the toxicity of the chemicals produced during the oxidation scan [9, 11]. Fast sweep rates, employed in fast cyclic voltammetry (FCV), provide near real-time analysis. A baseline curve must be obtained before testing begins to allow for the charging currents of the electrodes to be recorded and removed from the final results.

A neighboring electrode can be used for recording electrical signaling, or a grounded auxiliary system can provide the capability of recording both signals from the same electrode [12-14].

\section{DESCRIPTION AND FABRICATION}

The neural arrays consist of sixteen groups of three-electrode sites. Each group incorporates platinum working and auxiliary electrodes, and a chloridized silver reference electrode. A schematic of the array is shown in Figure 1. Minimum electrode dimensions ranged from 2 to $100 \mu \mathrm{m}$ on separate devices to allow the study of the effect of electrode size on performance. Individual site orientation was alternated. This allowed neighboring sites to be used in unison as a single larger electrode, providing additional information about the effect of electrode area on performance. 


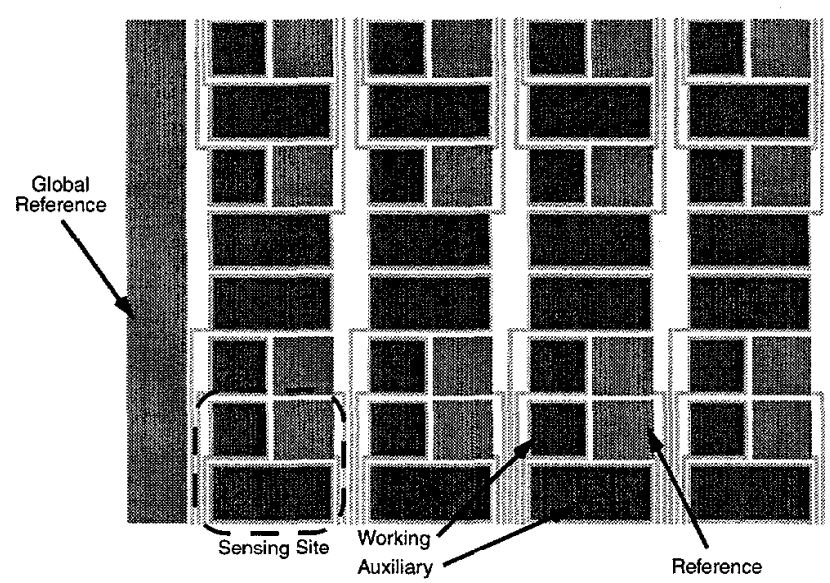

Figure 1. A schematic of the neural array. Site orientation is rotated to allow the combining of neighboring electrodes to study the effect of electrode size on performance.

Fabrication begins on standard $4 "<100>$ silicon substrates. A lower thermal oxide of $1.2 \mu \mathrm{m}$ provides electrical isolation. Platinum was used for interconnect due to its ability to serve as an excellent polarizable interface in solution. A modified lift-off procedure was used for patterning the interconnect. A timed etch in hydrofluoric acid "submerged" the area where the platinum was applied. This resulted in a nearly planar surface, improving the coverage of the top isolation layer. A $20 \mathrm{~nm}$ layer of titanium was used to help adhesion of the $100 \mathrm{~nm}$ platinum layer. Several sensor arrays were fabricated using a gold layer with platinum on the surface. It was hoped that the gold would provide devices with a lower resistance.

An isolation layer of $800 \mathrm{~nm}$ of PECVD silicon nitride was deposited over the platinum interconnect. A reactive ion etch was used to open the contacts. The resist for the silver lift-off step was then applied. However, before the silver was deposited, a second reactive ion etch was performed to etch $700 \mathrm{~nm}$ of the nitride layer. This left a shelf of nitride around the bottom of the silver sites as shown in Figure 2. In the past, our work with silver at these dimensions formed thin wall-like projections by coating the sidewalls of the lift-off resist. Later handling often caused these walls to collapse. The small pieces of silver left behind can move around on the device causing contamination of the platinum electrode surfaces. The etch helped to form a break line at the resist edge, preventing the formation of the walls. The etch was stopped short to prevent the formation of a seam between the silver sidewalls and the surrounding silicon nitride, since a seam might provide a route for encroachment of the test solution resulting in a dual contact.

Silver was applied $650 \mathrm{~nm}$ thick over a $20 \mathrm{~nm}$ layer of titanium as an adhesion promoter. The final lift-off was performed in

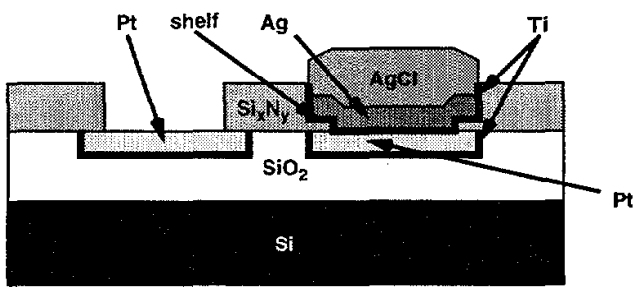

Figure 2. A cross-section of the completed passive array. Note the nitride shelf used to prevent solution from forming a dual contact to the electrode.

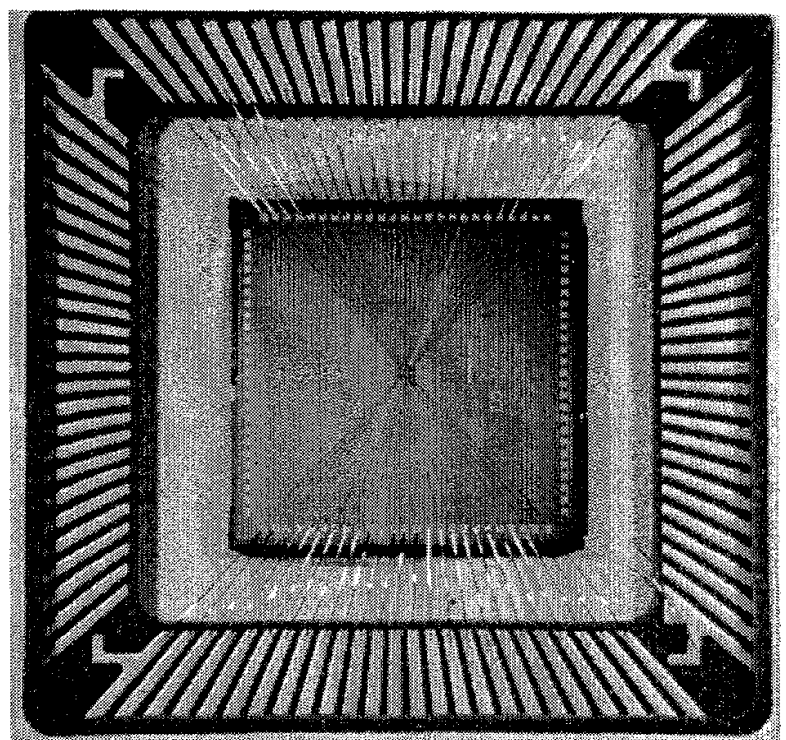

Figure 3. A packaged device wirehonded into ceramic pin grid array carrier before sealing with epoxy.

acetone. Early work using Microposit $1112 \mathrm{~A}$ photoresist remover (Shipley, Marlboro, MA, U.S.A.) caused discoloration of the silver reference electrodes. Acetone was less reactive to the silver; however, it lacks surfactants which prevent re-deposition of the silver. Care was required to insure all the waste metallization was completely removed in the lift-off process.

Finished chips were wire-bonded into ceramic 100-pin grid array packages (\#ECPG10020, Spectrum Semiconductor Materials, Inc., San Jose, CA, USA). The ceramic package provided both a high $\mathrm{I} / \mathrm{O}$ count for contact to the array and a large thermal mass. Cultured neurons are sensitive to thermal disturbances, and the ceramic package formed an adequate thermal buffer to allow the neuron cultures to be moved from an incubator into a test apparatus. Epoxy was used to insulate the wire bonds from the solution. Culture cylinders were then attached to the surface to provide conlainment of the culture medium. The resulting package system can be autoclaved for easy sterilization. A bonded device is shown in Figure 3 before epoxy sealing.

\section{RESULTS}

The culture cylinder was infused with neuron growth media for initial testing. After initial scans to establish the charging currents for the clectrode array, calibrated amounts of neurotransmitters were added to the media, and cyclic voltammograms were recorded. The temperature was carefully maintained by incubation to match neural culture conditions as closely as possible.

Three distinct peaks appeared in the dopamine calibration voltammograms. A peak at $500 \mathrm{mV}$ was related to dopamine concentration. Another peak with a potential of $>1 \mathrm{~V}$ may have been caused by the oxidation of the phenol red in the culture media. The other peak at $650 \mathrm{mV}$ is currently under investigation. Figure 4 shows the response of the $500 \mathrm{mV}$ dopamine peak and the $650 \mathrm{mV}$ peak, to increasing dopamine concentration. While the $500 \mathrm{mV}$ peak exhibits a linear dependence on dopamine concentration, the $650 \mathrm{mV}$ and $1 \mathrm{~V}$ peaks remain constant, demonstrating that the 


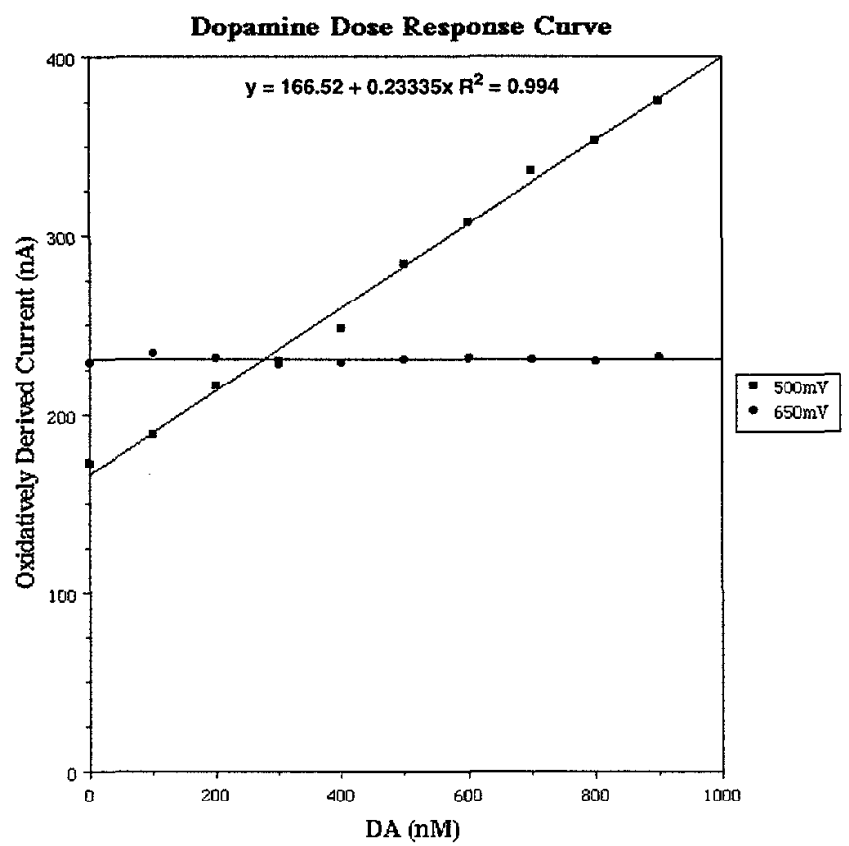

Figure 4. A dopamine calibration response curve. Notice that the neighboring $650 \mathrm{mV}$ peak is unaffected by dopamine concentration and represents a different electroactive compound in the culture media.

devices can distinguish dopamine from other electrochemically active species present in the culture media.

Live cell studies were performed with neuron cultures (hNT cell line from Stratagene, La Jolla, CA, U.S.A.). This cell line was chosen because it is known to be spontaneously active and requires no external stimulation to produce coordinated neural activity. These neurons release dopamine and acetylcholine.

Twenty-eight packaged neural sensor arrays were sterilized and prepared with cultures. After six days of incubation, 24 of the cultures were electrically active. The cultures were monitored for 75 days at which point the experiment was terminated.

For these preliminary tests, the internal reference electrode was replaced with a single external electrode. This simplified instrumentation by allowing the internal electrode to be used to detect neuroelectrical signals, but required all the sites to be swept in unison while performing cyclic voltammograms.

The results demonstrate the ability to detect neurotransmitter concentrations and to differentiate between dopamine and acetylcholine. The ability to concurrently record both neurochemical and neuroelectrical activity has been demonstrated (see Figure 5). Of particular note is the effect of the second group of spikes that apparently cause a release of neurotransmitter.

The devices have sufficient resolution to show the apparent interaction of a single action potential and the resulting neurotransmitter release (see Figure 6). Correlations between action potentials across the array were observed, showing the propagation of neural signals throughout the neural network.

The devices with platinum interconnect and those with a gold conductivity layer were both found to perform well. However, the pure platinum devices performed more closely to theoretical expectations. In the gold devices, it is suspected that the gold base layer and the platinum top-layer interdiffused. This could have occurred while the sealing epoxy was being cured in an oven.
Sensor Array N1F-8-3; Sensor \#14

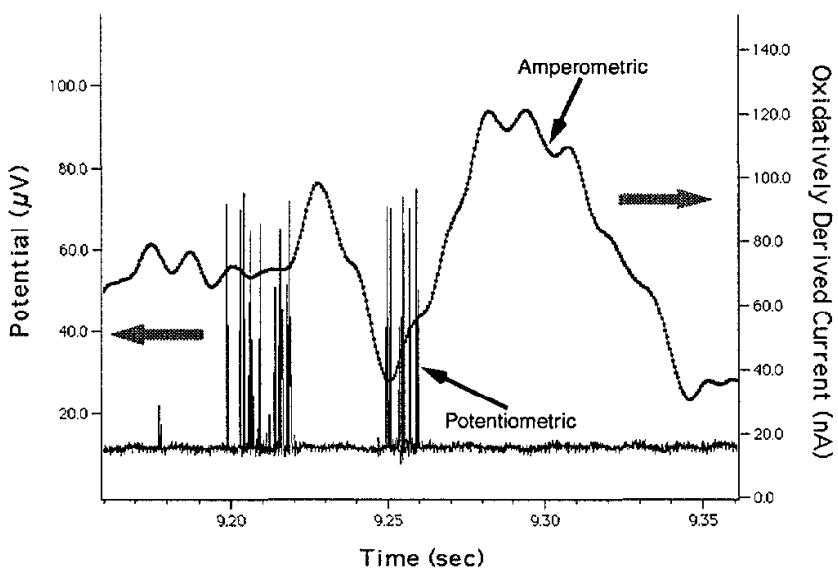

Figure 5. The combined chemical and electrical signal from a sensor site. The amperometric signal represents varying neurotransmitter concentrations; the potentiometric signal provides "spike" recording.

Diffusion between platinum and gold can occur at temperatures as low as $200^{\circ} \mathrm{C}$ [15]. Further evidence of this effect was observed when the resistance of the interconnect lines was measured. A distinct change in resistance was seen from an initial value to a higher value that stabilized with time. The current being applied to measure the resistance may have been high enough to cause the metals to interdiffuse.

An additional problem occurring on some devices was corrosion of the silver reference electrodes. This was observed on devices immediately after dicing, and is believed to be an electrolytic effect caused by the water used to cool the dicing saw. An electrolytic cell is formed between the large platinum bond pads and the smaller silver reference sites. Where a manufacturing defect broke the connection, no corrosion was seen. Future devices will incorporate a polymer layer to prevent this silver corrosion. The polymer can be easily removed with a solvent after dicing.

\section{Sensor Array N1F-8-3; Sensor \#0}

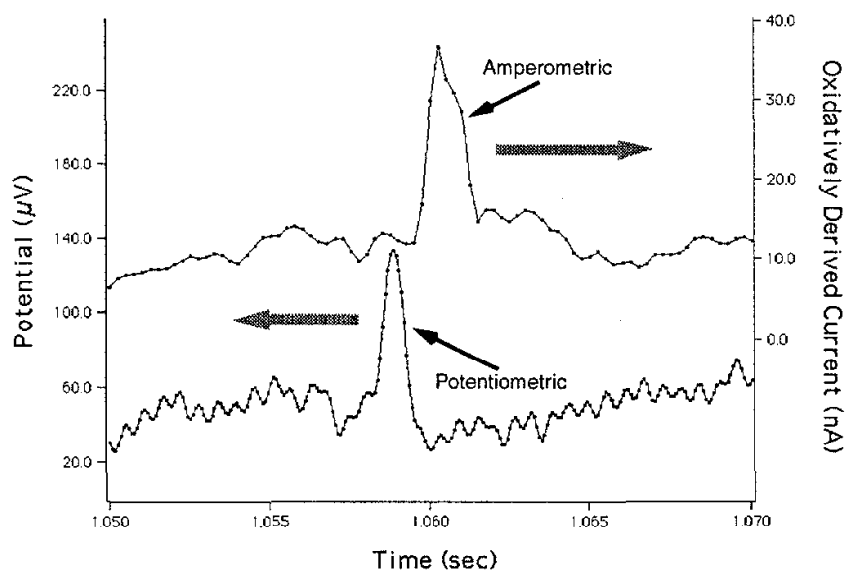

Figure 6. Superimposed potentiometric and amperometric traces. The amperometric signal appears to reveal the release. of neurotransmitter in response to the action potential shown in the potentiometric recording. 


\section{CONCLUSIONS}

These new devices provide an exciting advancement in neuroscience. Due to their fabrication using silicon processing techniques, they are relatively simple to produce in large quantities. Their performance is more consistent from device to device than the carbon fiber electrodes that have been used in previous studies. Unlike previous silicon neural devices, these arrays provide access to both neuroelectrical and neurochemical activity concurrently, providing the ability to study electrical and chemical interactions in neuronal communication. In addition, they provide the capability to study a system of neurons, allowing the study of cause-effect relationships. The use of cell culture provides the ability to study neural systems with no trauma to the tissue, such as that incurred by in vivo studies, and provides a long window of study, over 75 days.

The use of spontaneously active neurons eliminated the need for external stimulation of the culture. This reduces potential external influence on the neurons and improves the validity of the results. The silicon substrate allows for the incorporation of active electronics for amplification, noise reduction, and expansion of the array size while reducing lead counts.

The current, patent-pending, passive neural arrays hold a great deal of promise in future work, and research and development on them continues. Improvements in metallization are intended to remove remaining corrosion problems and more advanced lithography techniques will be applied to reduce the electrode size below $2 \mu \mathrm{m}$.

This work will allow neuroscientists to study the communication between neurons in much greater detail than was previously possible. Such research will enhance our understanding of the nervous system, and may provide valuable insights into a wide variety of neural disorders.

\section{ACKNOWLEDGEMENTS}

This work was supported by Advanced Sensor Technologies of Farmington Hills, Michigan, U.S.A., and NIH NINDS SBIR Grant \#1 R43 NS37989-01.

\section{REFERENCES}

1. K. D. Wise, J. B. Angell, A. Starr, "An Integrated-Circuit Approach to Extracellular Microelectrodes," IEEE Transactions on Biomedical Engineering, v. BME-17, n. 3, pp. 238-247, 1970

2. M. Kuperstien, D. A. Whittington, "A Practical 24 Channel Microelectrode for Neural Recording in Vivo," IEEE Transactions on Biomedical Engineering, v. BME-28, n. 3, pp. 288-293, 1981

3. N. A. Blum, B. G. Carkhuff, H. K. Charles, Ir., R. L. Edwards, R. A. Meyer, "Multisite Microprobes for Neural Recordings", IEEE Transactions on Biomedical Engineering, v. 38, n. 1, pp. $68-74,1991$.

4. P. Connolly, P. Clark, A. S. G. Curtis, J. A. T. Dow, C. D. W. Wilkinson, "An Extracellular Microelectrode Array for Monitoring Electrogenic Cells in Culture," Biosensors \& Bioelectronics, v. 5, pp. 223-234, 1990.

5. E. Valderrama, P. Garrido, E. Cabruja, P. Heiduschka, A. Harsch, W. Göpel, "Microfabrication and Characterization of Microelectrode Arrays for in Vivo Nerve Signal Recording," Proceedings of the 8th International Conference on Solid-State Sensors and Actuators, and Eurosensors IX, pp. 63-66, June 25-29, 1995.

6. S. L. BeMent, K. D. Wise, D. J. Anderson, K. Najafi, K. L. Drake, "Solid-State Electrodes for Multichannel Multiplexed Intracortical Neuronal Recording," IEEE Transactions on Biomedical Engineering, v. BME-33, n. 2, pp. 230-241 (1986).

7. C. Kim, K. D. Wise, "A 64-Site Multishank CMOS Low-Profile Neural Stimulating Probe," IEEE Journal of Solid-State Circuits, v. 31, n. 9, pp. 1230-1238 (1996).

8. J. Krüger, "Multiple Microelectrodes," Methods in Neurosciences: Electrophysilogy and Microinjection, v. 4, New York:Academic Press, pp. 249-265 (1991).

9. J. A. Stamford, Monitoring Neuronal Activity: A Practical Approach, IRL Press, Oxford, pp. 113-145, 1992.

10. A. J. Bard, L. R. Faulkner, Electrochemical Methods, New York:John Wiley \& Sons, pp. 22-26, 1980.

11. J. A. Stamford, "Fast cyclic voltammetry: measuring transmitter release in "real-time,", Journal of Neuroscience Methods, v. 34 pp. 67-72, 1990.

12. J. A. Stamford, "In Vivo Voltammetry," Methods in Neurosciences: Electrophysiology and Microinjection, v. 4, New York:Academic Press, pp. 127-141, 1991.

13. J. Millar, T. G. Barnett, "Basic instrumentation for fast cyclic voltammetry," Journal of Neuroscience Methods, v. 25, pp. 91-95, 1998.

14. J. A. Stamford, P. Palij, C. Davidson, C. M. Jorm, J. Millar, 'Simultaneous 'real-time' electrochemical and electrode physiological recording in brain slices with a single carbon-fibre microelectrode," Journal of Neuroscience Methods, v. 50, pp. 279-290, 1993.

15. R. Belser, "Alloying Behavior of Thin Bi-metal Films, Simultaneously or Successively Deposited," Journal of Applied Physics, v. 31, n. 3 , pp. $562-570,1960$. 